\section{Genetic and Metabolic Disease in Pediatrics}

Butterworth Internationai Medical Reviews, Pediatrics Volume 5. Edited by June $\mathrm{K}$ Lloyd and Charles R Scriver. (Pp 324; figures + tables. $£ 45 \cdot 00$.) London: Butterworth. 1985.

Most genetic and metabolic diseases are apparent at birth, are detectable before or soon after birth, or become evident during childhood. There are important exceptions which do not become evident until adulthood.

In this book inherited structural and functional disorders have been chosen by the editors and their 14 contributors to illustrate the interplay of genotype and environment which produces the many and varied disease phenotypes described in paediatric practice. Although some of the examples are individually rare this has been skilfully achieved by keeping homeostasis as a main theme.

In an excellent introduction the editors explain in a chapter entitled 'Genetic causes of deviant metabolism. What do we know? What can we do?' their reasons for choosing the subsequent 11 chapters to illustrate their homeostatic theme. Marcus Pembrey and Robert S Sparkes, in workmanlike and lucid accounts of genes, their anatomy, biochemistry, function, and anomalies, provide for the nongeneticist a helpful introduction to the language and techniques of the geneticist. In chapter 4 , Byers and Bonadio give a fascinating and up to the moment account of the molecular basis of clinical heterogeneity in osteogenesis imperfecta by reference to studies of inherited disorders of type I collagen. The next four chapters deal with the molecular basis for abnormal lysosomal enzyme transport in mucolipidoses II and III, the lactic acidaemias, defects in urea synthesis, and the hyperphenylalaninaemias. All give excellent individual accounts of the disorders and illustrate how such defects affect the body's homeostatic mechanisms. These chapters, together with those on the hyperlipidaemias, maternal-fetal metabolic homeostasis, maternal phenylketonuria, and androgen receptor disorders, well illustrate the intricate interplay between environment and genetic endowment in the maintenance of constancy in the body's 'milieu interior'.

I can recommend this volume to all geneticists with an interest in congenital abnormalities and metabolic disorders and all paediatricians who would wish to understand better their genetic basis, together with the biochemists who deal with metabolic disorders, geneticists, and paediatricians. This "evolutionary view of disease in man" raises issues beyond those of prevention and therapy of specific disorders, including moral and social issues. For these reasons I would recommend this book to all who wish to understand better the basis for a healthy life in metabolic equilibrium and the function, if not the origin, of our species.

FORRESTER COCKBURN

\section{An Introduction to Recombinant DNA}

By A E H Emery. (Pp x + 223; figures + tables. £6-75.) Chichester: Wiley. 1984.

Doctors are notorious for using two long words when a single short one would suffice. Molecular biology has gone even further by devising a new language of its own, which for the uninitiated makes a difficult subject almost incomprehensible. Thus, it is a pleasure to encounter a book which removes much of the mystery and mystique from a subject which promises to revolutionise medicine.

This lucid and concise text covers all aspects of molecular genetics in 10 short succinct chapters. The first sets the scene with an historical background and is followed by an outline of the structure of DNA. The technology for handling DNA is covered in chapter 3 while chapter 4 moves on to a consideration of the structure and function of the genome.

Chapters 5 and 6 are devoted to a review of the molecular pathology of Mendelian and multifactorial disorders and, in conjunction with chapter 7 which discusses the existing and potential applications of these techniques in clinical genetics, will be of particular interest to those involved in genetic counselling. The concluding chapters provide an overview, occasionally speculative, of the possible applications of the 'new genetics' in the broader fields of gene therapy, agriculture, and the study of evolution. The moral and ethical aspects of these developments are also considered.

Each chapter is well illustrated with line drawings and is followed by an appropriate bibliography. The book is completed by a particularly valuable glossary and comprehensive index. This well written and remarkably inexpensive book will be much appreciated by students and practitioners of all branches of medicine and can be highly recommended. This will undoubtedly be the first of many editions.

I D Young

Basic Cloning Techniques. A Manual of Experimental Procedures

Edited by R H Pritchard and I B Holland. (Figures + tables. £11.80.) Oxford: Blackwell Scientific Publications. 1985.

There is currently no shortage of practical textbooks 
on recombinant DNA methodology. This one is based on a practical course run by the University of Leicester and contains five detailed articles: Mammalian cDNA cloning; Analysis of nucleic acids (restriction maps, Southern and Northern blots); Systems for the analysis of gene expression; Cloning in phage lambda; and electron microscopy of nucleic acids. Thus, it covers some areas that are not dealt with in other widely used texts such as that of Maniatis et al. The strength of this book is the large amount of practical detail that is included, especially useful for beginners in this field. Its coverage is limited to the five topics listed above and would therefore need to be supplemented by other articles. However, no attempt is made to guide the reader to any of this material, and in general the references given are rather few in number and not particularly up to date. For example, in the chapter on lambda cloning there is no mention of the recently developed and extremely useful EMBL series of vectors.

I would suggest that potential buyers have a good look at the book before they commit themselves, and be aware that more up to date procedures are available in many cases.

Duncan Shaw

\section{Gene Expression During Normal and Malignant Differentiation}

Edited by L C Andersson, C G Gahmberg, and P Ekblom. (Pp 237; figures + tables. £26.50.) Florida: Academic Press. 1985.

As described in the preface, this book comprises the highlights of a symposium held in 1984. The symposium was intended to cover the genetic regulation of cellular functions from diverse positions. The combination of an all-encompassing title and a limited number of contributions has selfevident inherent problems which have not been entirely overcome. To satisfy the bias of current interest in molecular biology, eight of the 18 chapters deal with the effects of viral and cellular oncogenes. These are overall fairly informative articles, dealing with oncogene activation, expression in normal and neoplastic cells, phosphorylation, similarity of oncogene products and growth factors, HTLV, and so forth, but their number tends somewhat to compress illustrations of other viewpoints. Representations of these include influence of cell-cell interactions and the architectural features of the cell ('Cells as tensegrity structures') on differentiation, various aspects of induction and control of differentiation, and patterns of membrane glycoproteins and glycolipids, associated with differentiation and proliferation.

It is highly unlikely that specialists will find anything in the relevant chapters with which they will be unfamiliar. Anything which fulfilled the criterion of 'very recent developments in biomedical research' at the time of the symposium has since been published in far more detail in the refereed journals. The book may be of interest to the specialist who is curious about advances and approaches in related areas but does not expect to expand his knowledge extensively. It would, perhaps, be of more value to the novice not yet committed, who needs a wide selection from which to make his choice.

There is no uniformity of presentation of the chapters, whether regarding structure, typescript (the volume has been produced by 'rapid manuscript reproduction' which does not account for the number of typographical errors in the list of contents), or length. Some, therefore, contain much information, whereas others may be described as brusque. This, however, may relate to the specialisation of the reader. Most tend towards the style of the mini-review or overview, while few assume the format of the scientific paper. These inconsistencies serve to emphasise the lack of cohesion in the book.

Peter Davies

\section{Genetic Engineering-Principles and Methods}

Volume 7. Edited by J K Setlow and A Hollaender. (Pp 359; figures + tables. \$52.50.) New York: Plenum Press. 1985.

The seventh volume of the series 'Genetic Engineering-Principles and Methods' contains 15 chapters on a wide variety of biological topics. These range from mainly methodological contributions, such as use of DNA expression libraries, computer analysis of DNA sequences, SP6 RNA polymerase reactions, and gene transfer using retroviral vectors, to reviews on some applications of DNA technology, including DNA replication and transcription, plant molecular genetics, bacterial pathogenicity, cytomegaloviruses, gene therapy, bacterial catabolic plasmids, and DNA markers for human genetic disorders. The more practically orientated chapters contain much useful experimental detail and all of the contributions are well written and presented, with sufficient references given to allow particular points to be pursued further. As a whole, the book is a worthwhile 